\title{
Le congruenze.
}

(Di 'T. Cifarelli, a Napoli.)

1. Definiremo con Kummer analiticamente una congruenza esprimendo le coordinate $x, y, z$ di un punto di un raggio, ed i suoi coseni direttori $X, Y, Z$ in funzione di due variabili indipendenti $u, v$. Supporremo che le sei funzioni $x, y, z, X, Y, Z$ siano finite e continue insieme alle loro derivate parziali. Si troverà quindi il punto $(x, y, z)$ sopra una certa superficie, ed ogni altro punto di un raggio sarà definito mediante la sua distanza (ascissa) dal punto di partenza $(x, y, z)$.

Conducendo per l'origine delle coordinate i raggi paralleli alle direzioni positive di quelli della congruenza, e tagliandoli con la sfera $x^{2}+y^{2}+z^{2}=1$ avremo la rappresentazione sferica della congruenza.

Consideriamo le seguenti dodici funzioni fondamentali :

$$
\begin{array}{ccc}
\Sigma\left(\frac{\partial X}{\partial u}\right)^{2}=E, & \Sigma \frac{\partial X}{\partial u} \frac{\partial X}{\partial v}=F, & \Sigma\left(\frac{\partial X}{\partial v}\right)^{2}=G, \\
\Sigma \frac{\partial X}{\partial u} \frac{\partial x}{\partial u}=e, & \Sigma \frac{\partial X}{\partial u} \frac{\partial x}{\partial v}=f, \quad \Sigma \frac{\partial X}{\partial v} \frac{\partial x}{\partial u}=f^{\prime}, & \Sigma \frac{\partial X}{\partial v} \frac{\partial x}{\partial v}=g, \\
\Sigma\left(\frac{\partial x}{\partial u}\right)^{2}=\mathbf{E}, & \Sigma \frac{\partial x}{\partial u} \frac{\partial x}{\partial v}=\mathbf{F}, & \Sigma\left(\frac{\partial x}{\partial v}\right)^{2}=\mathbf{G}, \\
& \Sigma X \frac{\partial x}{\partial u}=1, \quad \Sigma X \frac{\partial x}{\partial v}=\mathbf{B} ;
\end{array}
$$

di cui le $(a),(b),(c)$ servono rispettivamente a definire le tre forme differenziali quadratiche:

$$
\begin{aligned}
d \sigma^{2}=\Sigma d X^{2} & =E d u^{2}+2 F d u d v \quad+G d v^{2} \\
\Sigma d X d x & =e d u^{2}+\left(f+f^{\prime}\right) d u d v+g d v^{2} \\
d s^{2}=\Sigma d x^{2} & =\mathbf{E} d u^{2}+2 \mathbf{F} d u d v \quad+\mathbf{G} d v^{2}
\end{aligned}
$$


mentre le $(d)$ rappresentano delle quantità proporzionali ai coseni degli angoli che i raggi della congruenza formano con le linee coordinate sulla superficie di partenza. Chiameremo le tre forme differenziali quadratiche rispettivamente prima, seconda e ter'za; e notiamo che la prima dà il quadrato dell'elemento lineare sull'immagine sferica, o, ciò che è lo stesso, l'angolo di due generatrici successive, mentre l'ultima indica il quadrato dell'elemento lineare sulla superficie $(x, y, z)$; queste due sono quindi sempre definite.

2. Ricorderemo in questo paragrafo alcune formole e proprietà relative alla sfera. Le funzioni $(a),(b),(c),(d)$ riduconsi a tre : $E, F, G$; essendo uguali quelle rappresentate dalle stesse lettere, ed annullandosi le $(d)$. Indicando con $\left\{\begin{array}{c}r s \\ t\end{array}\right\}$ i nuovi simboli di Christoffred relativi alla prima forma fondamentale $\left(^{*}\right)$ si hanno le seguenti importanti relazioni :

$$
\begin{aligned}
& \frac{\partial^{2} X}{\partial u^{2}}=\left\{\begin{array}{c}
1 \\
1
\end{array}\right\} \frac{\partial X}{\partial u}+\left\{\begin{array}{c}
1 \\
2
\end{array}\right\} \frac{\partial X}{\partial v}-E X, \\
& \frac{\partial^{2} X}{\partial u \partial v}=\left\{\begin{array}{c}
1 \\
1 \\
1
\end{array}\right\} \frac{\partial X}{\partial u}+\left\{\begin{array}{c}
1 \\
2
\end{array}\right\} \frac{\partial X}{\partial v}-F X, \\
& \frac{\partial^{2} X}{\partial v^{2}}=\left\{\begin{array}{c}
22 \\
1
\end{array}\right\} \frac{\partial X}{\partial u}+\left\{\begin{array}{c}
22 \\
2
\end{array}\right\} \frac{\partial X}{\partial v}-G X,
\end{aligned}
$$

con le analoghe per $Y$ e $Z$. Applicando a queste le condizioni d'integrabilità si vede che i tre coefficienti $E, F, G$ non sono tra loro indipendenti, ma bensì legati dalla seguente relazione dovuta a GıJss:

$$
\begin{gathered}
\frac{1}{2 \sqrt{E G-F^{2}}}\left\{\frac{\partial}{\partial u}\left[\frac{F}{E \sqrt{E G-F^{2}}} \frac{\partial E}{\partial v}-\frac{1}{\sqrt{E G-F^{2}}} \frac{\partial G}{\partial u}\right]+\right. \\
\left.+\frac{\partial}{\partial v}\left[\frac{2}{\sqrt{E G-F^{2}}} \frac{\partial F}{\partial u}-\frac{1}{\sqrt{E G-F^{2}}} \frac{\partial E}{\partial v}-\frac{F}{E \sqrt{E G-F^{2}}} \frac{\partial E}{\partial u}\right]\right\}=+1 .
\end{gathered}
$$

Questa relazione necessaria è anche sufficiente, e si ha il seguente teorema :

Data una forma differenziale quadratica definita

$$
\varphi=E d u^{2}+2 F d u d v+G d v^{2}
$$

affinchè esista una sfera di raggio uguale all'unità che l'ammetta come sua

(*) Tutto ció che è detto in questo paragrafo è tolto dalla Geometria differenziale del prof. Bianchi. Cosi anche in seguito per qualungne richiamo alla teoria delle superflicie ricorrero al defto trattato. 
forma fondamentale, è necessario e sufficiente che sia verificata la condizione di Gauss.

3. Cio posto si potranno sempre determinare sei coefficienti incogniti $\alpha, \beta, \gamma, \alpha_{1}, \beta_{1}, \gamma$ in modo da avere:

$$
\begin{array}{ll}
\frac{\partial x}{\partial u}=\alpha \frac{\partial X}{\partial u}+\beta \frac{\partial X}{\partial v}+\gamma X, & \frac{\partial x}{\partial v}=\alpha_{1} \frac{\partial X}{\partial u}+\beta_{1} \frac{\partial X}{\partial v}+\gamma_{1} X, \\
\frac{\partial y}{\partial u}=\alpha \frac{\partial Y}{\partial u}+\beta \frac{\partial Y}{\partial v}+\gamma Y, & \frac{\partial y}{\partial v}=\alpha_{1} \frac{\partial Y}{\partial u}+\beta_{1} \frac{\partial Y}{\partial v}+\gamma_{1} Y, \\
\frac{\partial z}{\partial u}=\alpha \frac{\partial Z}{\partial u}+\beta \frac{\partial Z}{\partial v}+\gamma Z, & \frac{\partial z}{\partial v}=\alpha_{1} \frac{\partial Z}{\partial u}+\beta_{1} \frac{\partial Z}{\partial v}+\gamma_{1} Z,
\end{array}
$$

perchè il comune determinato $\sqrt{E G-F^{2}}$ è diverso da zero. Perciò moltiplicandole rispettivamente prima per $\frac{\partial X}{\partial u}, \frac{\partial I}{\partial u}, \frac{\partial Z}{\partial u}$, poi per $\frac{\partial X}{\partial v}, \frac{\partial Y}{\partial v}, \frac{\partial Z}{\partial v}$, ed infine per $X, Y, Z$, ed ogni volta sommando si ha:

ossia :

$$
\begin{array}{ll}
e=\alpha E+\beta F, & f=\alpha_{1} E+\beta_{1} F, \\
f^{\prime}=\alpha F+\beta G, & g=\alpha_{1} F+\beta_{1} G, \\
\mathbf{A}=\gamma, & \boldsymbol{B}=\gamma_{1} ;
\end{array}
$$

$$
\left.\begin{array}{lll}
\alpha=\frac{e G-f^{\prime} F}{E G-F^{2}}, & \beta=\frac{f^{\prime} E-e F}{E G-F^{2}}, & \gamma=A, \\
\alpha_{1}=\frac{f G-g F}{E G-F^{2}}, & \beta_{1}=\frac{g E-f F}{E G-F^{2}}, & \gamma_{1}=1 .
\end{array}\right\}
$$

Ricavate in tal modo le funzioni $\alpha, \beta, \gamma, \alpha_{1}, \beta_{1}, \gamma_{1}$ in funzione delle $(a),(b),(d)$, possiamo subito esprimere le $(c)$ mediante queste. In vero moltiplicando le (2) rispettivamente per $\frac{\partial x}{\partial u}, \frac{\partial y}{\partial u}, \frac{\partial z}{\partial u}$ e poi per $\frac{\partial x}{\partial v}, \frac{\partial y}{\partial v}, \frac{\partial z}{\partial v}$ ed ogni volta sommando si ha:

$$
\left.\begin{array}{l}
\mathbf{E}=e \alpha+f^{\prime} \beta+\gamma^{2}, \\
\mathbf{F}=f \alpha_{1}+g \beta+\gamma \gamma_{1}=e \alpha_{1}+f^{\prime} \beta_{1}+\gamma \gamma_{1}, \\
\mathbf{G}=f \alpha_{1}+g \beta_{1}+\gamma_{1}^{2} .
\end{array}\right\}
$$

Adunque per le (3) si scorge che le tre funzioni che definiscono l'elemento lineare della superficie $(x, y, z)$ si sanno in termini finiti calcolare mediante le altre $(a),(b),(d)$. Noi supporremo date queste nove funzioni, ciò̀ 
supporremo che assieme alle prime due forme differenziali quadratiche sieno date le due funzioni $\mathbf{A}$ e $\mathbf{B}$.

4. Si è visto che per l'esistenza di $X, Y, Z$ i coefficienti della prima forma differenziale quadratica devono soddisfare all'equazione alle derivate parziali (I), e che anzi quella è pure condizione sufficiente. Ammesso ora soddisfatta la (I), scriviamo le condizioni d'integrabilità delle (2):

e l'analoghe per $y$ e $z$.

$$
\frac{\partial}{\partial v} \frac{\partial x}{\partial u}=\frac{\partial}{\partial u} \frac{\partial x}{\partial v}
$$

Sviluppando col tener conto delle (1) si trovano le condizioni :

$$
\begin{aligned}
& \frac{\partial \alpha}{\partial v}-\frac{\partial \alpha_{1}}{\partial u}-\alpha_{1}\left\{\begin{array}{c}
1 \\
1
\end{array}\right\}+\left(\alpha-\beta_{1}\right)\left\{\begin{array}{c}
12 \\
1
\end{array}\right\}+\beta\left\{\begin{array}{c}
22 \\
1
\end{array}\right\}-\mathbf{B}=0,
\end{aligned}
$$

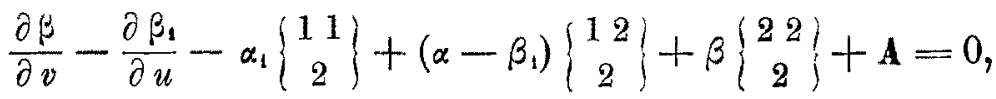

$$
\begin{aligned}
& \frac{\partial \mathbf{A}}{\partial v}-\frac{\partial \mathbf{B}}{\partial u}+\alpha_{1} E-\left(\alpha-\beta_{1}\right) F-\beta G=0,
\end{aligned}
$$

che si trasformano agevolmente nelle altre:

$$
\begin{aligned}
& \frac{\partial e}{\partial v}-\frac{\partial f}{\partial u}-\left\{\begin{array}{c}
1 \\
1
\end{array}\right\} e+\left\{\begin{array}{c}
1 \\
1
\end{array}\right\} f-\left\{\begin{array}{c}
12 \\
2
\end{array}\right\} f^{\prime}+\left\{\begin{array}{c}
1 \\
2
\end{array}\right\} g+F \mathbf{A}-E \mathbf{B}=0 \\
& \frac{\partial f^{\prime}}{\partial v}-\frac{\partial g}{\partial u}-\left\{\begin{array}{c}
22 \\
1
\end{array}\right\} e+\left\{\begin{array}{c}
1 \\
1
\end{array}\right\} f-\left\{\begin{array}{c}
22 \\
2
\end{array}\right\} f^{\prime}+\left\{\begin{array}{c}
12 \\
2
\end{array}\right\} g+G \mathbf{A}-F \mathbf{B}=0 \\
& \frac{\partial \mathbf{A}}{\partial v}-\frac{\partial \mathbf{B}}{\partial u}+f-f^{\prime}=0
\end{aligned}
$$

Sono queste condizioni necessarie per l'esistenza delle funzioni $x, y, z$. Sicchè, data una congruenza, le nove funzioni fondamentali $(a),(b),(d)$ non sono tra loro indipendenti, ma devono soddisfare alle quattro condizioni (I) e (II) date dal prof. Cessìno per le congruenze (*). Di esse la (I) e le prime due delle (II) per le congruenze normali si riducono alle condizioni del CoDAZzi, mentre l'ultima riesce identicamente soddisfatta, come fra breve vedremo. Le (II) sono anche sufficienti, perchè posto:

$$
d x=\left(\alpha \frac{\partial X}{\partial u}+\beta \frac{\partial X}{\partial v}+\gamma X\right) d u+\left(\alpha_{1} \frac{\partial X}{\partial u}+\beta_{1} \frac{\partial X}{\partial v}+\gamma_{1} X\right) d v
$$

(*) Geometria intrinseca, pag. 205 . 
con l'analoghe per $d y$ e $d z$, e supposta verificata la (I), i coefficienti di $d u$ e $d v$ sono funzioni conosciute di $u$ e di $v$, ed affinchè si abbiano a secondo membro dei differenziali esatti si trovano per equazioni di condizione proprio le (II) che noi supponiamo verificate. Si avrà perciò integrando:

$$
\begin{array}{cc}
x=\int\left[\left(\alpha \frac{\partial X}{\partial u}+\beta \frac{\partial X}{\partial v}+\gamma X\right) d u+\left(\alpha_{1} \frac{\partial X}{\partial u}+\beta_{1} \frac{\partial X}{\partial v}+\gamma_{1} X\right) d v\right], & z=\cdots \cdots,
\end{array}
$$

le quali definiscono un'unica superficie $(x, y, z)$, a meno uno spostamento rigido nello spazio. Facendo poi passare per ciascun punto $(u, v)$ di tale superficie un raggio di coseni direttori $X, Y, Z$ unicamente definito dall'immagine sferica sarà individuata un' uniea congruenza relativa alle funzioni $(a),(b),(d)$ soddisfacenti alle condizioni (I) e (II). Si ha dunque il teorema:

Date arbitrariamente le nove funzioni $(a),(b),(d)$, di cui $(a)$ e $(b)$ siano $i$ coefficienti di due forme differenziali quadratiche, affinchè vi corrisponda una congruenza che le abbia come funzioni fondamentali, è necessario e sufficiente che esse soddisfino alle quattro equazioni alle derivate parziali (I) $e$ (II); di queste la (I) ci fornirà un' unica immagine sferica, e mediante quadrature si ricaverà (a meno uno spostamento rigido nello spazio), per. ogni punto della immagine un unico punto $(x, y, z)$ per cui passerà il raggio di data immagine.

5. Derivando le $(b)$, tenendo conto delle (1) e della condizione d'integrabilita $\frac{\partial}{\partial u} \frac{\partial}{\partial v}=\frac{\partial}{\partial v} \frac{\partial}{\partial u}$, si ha il seguente gruppo di formole notate da Wetngarten $(*)$ per le superficie:

$$
\left.\begin{array}{l}
\frac{\partial e}{\partial u}=\left\{\begin{array}{c}
1 \\
1
\end{array}\right\} e+\left\{\begin{array}{c}
1 \\
2
\end{array}\right\} f^{\prime}-E \mathbf{A}+\Sigma \frac{\partial X}{\partial u} \frac{\partial^{2} x}{\partial u^{2}}, \\
\frac{\partial e}{\partial v}=\left\{\begin{array}{c}
1 \\
1
\end{array}\right\} e+\left\{\begin{array}{c}
12 \\
2
\end{array}\right\} f^{\prime}-F \mathbf{A}+\Sigma \frac{\partial X}{\partial u} \frac{\partial^{2} x}{\partial u \partial v}, \\
\frac{\partial f}{\partial u}=\left\{\begin{array}{c}
1 \\
1
\end{array}\right\} f+\left\{\begin{array}{c}
11 \\
2
\end{array}\right\} g-E \mathbf{B}+\Sigma \frac{\partial X}{\partial u} \frac{\partial^{2} x}{\partial u \partial v}, \\
\frac{\partial f}{\partial v}=\left\{\begin{array}{c}
12 \\
1
\end{array}\right\} f+\left\{\begin{array}{c}
12 \\
2
\end{array}\right\} g-F \mathbf{B}+\Sigma \frac{\partial X}{\partial u} \frac{\partial^{2} x}{\partial v^{2}},
\end{array}\right\}
$$

(*) Veggasi Bravohi, Geom. diff., pag. 123.

Annali di Matematica, Serie III, tomo II. 


$$
\begin{aligned}
& \frac{\partial f^{\prime}}{\partial u}=\left\{\begin{array}{c}
12 \\
1
\end{array}\right\} e+\left\{\begin{array}{c}
12 \\
2
\end{array}\right\} f^{\prime}-F \mathbf{A}+\Sigma \frac{\partial X}{\partial v} \frac{\partial^{2} x}{\partial u^{2}}, \\
& \frac{\partial f^{\prime}}{\partial v}=\left\{\begin{array}{c}
22 \\
1
\end{array}\right\} e+\left\{\begin{array}{c}
22 \\
2
\end{array}\right\} f^{\prime}-G \mathbf{A}+\Sigma \frac{\partial X}{\partial v} \frac{\partial^{2} x}{\partial u \partial v}, \\
& \frac{\partial g}{\partial u}=\left\{\begin{array}{c}
12 \\
1
\end{array}\right\} f+\left\{\begin{array}{c}
12 \\
2
\end{array}\right\} g-F \mathbf{B}+\Sigma \frac{\partial X}{\partial v} \frac{\partial^{2} x}{\partial u \partial v}, \\
& \frac{\partial g}{\partial v}=\left\{\begin{array}{c}
22 \\
1
\end{array}\right\} f+\left\{\begin{array}{c}
22 \\
2
\end{array}\right\} g-G \mathbf{B}+\Sigma \frac{\partial X}{\partial v} \frac{\partial^{2} x}{\partial v^{2}},
\end{aligned}
$$

Se ora poniamo:

viene :

$$
\mathbf{A}_{1}=\Sigma x \frac{\partial X}{\partial u}, \quad \mathbf{B}_{1}=\Sigma x \frac{\partial X}{\partial v}, \quad W=\Sigma x X
$$

$$
\mathbf{A}+\boldsymbol{\Lambda}_{1}=\frac{\partial W}{\partial u}, \quad \mathbf{B}+\mathbf{B}_{1}=\frac{\partial W}{\partial v}
$$

e derivando le funzioni $\mathbf{A}, \mathbf{B}, \mathbf{A}_{1}, \mathbf{B}_{1}$ abbiamo quest'altre formole:

$$
\begin{aligned}
& \frac{\partial \mathbf{A}}{\partial u}=e+\Sigma X \frac{\partial^{2} x}{\partial u^{2}}, \\
& \frac{\partial \mathbf{B}}{\partial u}=f+\Sigma X \frac{\partial^{2} x}{\partial u \partial v}, \\
& \frac{\partial \mathbf{A}_{1}}{\partial u}=e+\left\{\begin{array}{c}
11 \\
1
\end{array}\right\} \mathbf{A}_{1}+\left\{\begin{array}{c}
11 \\
2
\end{array}\right\} \mathbf{B}_{1}-E W, \\
& \frac{\partial \mathbf{B}_{1}}{\partial u}=f^{\prime}+\left\{\begin{array}{c}
12 \\
1
\end{array}\right\} \mathbf{A}_{1}+\left\{\begin{array}{c}
12 \\
2
\end{array}\right\} \mathbf{B}_{1}-F W, \\
& \frac{\partial \mathbf{A}}{\partial v}=f+\Sigma X \frac{\partial^{2} x}{\partial u \partial v}, \\
& \frac{\partial \mathbf{B}}{\partial v}=g+\Sigma X \frac{\partial^{2} x}{\partial v^{2}}, \\
& \frac{\partial \mathbf{A}_{1}}{\partial v}=f+\left\{\begin{array}{c}
12 \\
1
\end{array}\right\} \mathbf{A}_{1}+\left\{\begin{array}{c}
12 \\
2
\end{array}\right\} \mathbf{B}_{1}-F W, \\
& \frac{\partial \mathbf{B}_{1}}{\partial v}=g+\left\{\begin{array}{c}
22 \\
1
\end{array}\right\} \mathbf{A}_{1}+\left\{\begin{array}{c}
22 \\
2
\end{array}\right\} \mathbf{B}_{1}-G W .
\end{aligned}
$$

Combinando opportunamente le (5) e le (7) si ritrovano le (II). Per quest'ultime la terza delle (II) può scriversi anche cos̀̀ :

$$
\frac{\partial \mathbf{A}_{1}}{\partial v}-\frac{\partial \mathbf{B}_{1}}{\partial u}+f^{\prime}-f=0 \text {. }
$$


6. Affinchè la congruenza sia normale su ogni raggio dev' esserci un punto almeno $(\xi, \eta, \xi)$ per il quale si abbia $\Sigma X d \xi=0$. Ora posto:

$$
\xi=x+t X, \quad \eta=y+t Y, \quad \zeta=z+t Z
$$

risulta :

$$
d t=-(\mathbf{A} d u+\mathbf{B} d v),
$$

e la condizione d'integrabilità :

$$
\frac{\partial \mathbf{A}}{\partial v}=\frac{\partial \mathbf{B}}{\partial u},
$$

ei dà :

$$
f=f^{\prime} .
$$

Inversamente se è $f=f^{\prime}$ si ricava per la terza delle (II) la (9), e s'otterrà, integrando, una famiglia di superficie parallele ed ortogonali alla congruenza. Tenendo conto delle (9) e (10) si vede che la terza delle (II) riesce identicamente nulla, mentre le prime due si riducono ad altre ancora più generali di quelle del Codazzr, perchè la congruenza è legata ad una superficie arbitraria che la taglia. Per ottenere effettivamente quelle del Codazzr bisogna supporre $t=$ cost. ossia $\mathbf{A}=\mathbf{B}=0$, e sostituire ai coefficienti $(a)$ le loro espressioni in funzione di $(b)$ e $(c)$.

$\grave{\mathrm{E}}$ anche $\mathbf{A}_{1} d u+\mathbf{B}_{1} d v$ un differenziale esatto, e guardando $\mathrm{i}$ due differenziali esatti :

$$
\begin{aligned}
& \mathbf{A} d u+\mathbf{B} d v=X d x+Y d y+Z d z=-d t, \\
& \mathbf{A}_{1} d u+\mathbf{B}_{1} d v=x d X+y d Y+z d Z=d(W+t),
\end{aligned}
$$

conseguenza l'un dell'altro, e caratterizzanti le congruenze normali, abbiano rispettivamente le seguenti interpretazioni meccaniche.

Nel moto di un punto sopra una superficie $(x, y, z)$, sollecitato da una forza costante $(X, Y, Z)$, si verifica l'integrale delle forze vive solo se l'insieme delle linee d'azione della forza che è applicata al punto in ogni istante costituisce una congruenza normale.

La funzione delle forze rappresenta la distanza tra i punti corrispondenti (sullo stesso raggio) di una superficie $\Sigma$ cui la congruenza è normale e della 
superficie $S \equiv(x, y, z)$. Le superficie di livello tagliano $S$ lungo le linee in cui tale distanza $t$ è costante.

Il secondo differenziale esatto dice che nel moto di un punto sopra una sfera $(X, Y, Z)$ si verifica l'integrale delle forze vive solo se, interpretando le componenti della forza direttamente applicata $(x, y, z)$ come coordinate di punti di una certa superficie $S$, e conducendo per $i$ punti di $S$ le rette parallele alle normali nei punti corrispondenti sulla sfera, si ha una congruenza normale.

La funzione delle forze rappresenta la distanza dell'origine (centro della sfera) al piano tangente ad una superficie $\Sigma$. Le superficie di livello $W+t=$ cost. tagliano la sfera lungo linee che hanno sulla superficie pedale di una $\Sigma$, rispetto all'origine come polo, anche linee sferiche come corrispondenti.

7. Si è visto che data una superficie $S$ la congruenza delle sue normali ha $f=f^{\prime}, \mathbf{\Lambda}=\mathbf{B}=0$. I nove coefficienti riduconsi dunque a 6 che sono $E, F, G$, e gli altri tre $e, f, g$ che s'usano rappresentare con - D, $-\mathbf{D}^{\prime},-\mathbf{D}^{\prime \prime}$. Ai primi tre si possono sempre sostituire gli altri $\mathbf{E}, \mathbf{F}, \mathbf{G}$ medianie le (4). Vogliamo ora calcolarci gli altri tre coefficienti $\mathbf{D}, \mathbf{D}^{\prime}, \mathbf{D}^{\prime \prime}$ relativi ad una superficie $S$ quando è nota una congruenza qualsiasi uscente da essa.

Si possono sempre determinare nove coefficienti incogniti: $a, b, c$; $a^{\prime}, b^{\prime}, c^{\prime} ; a^{\prime \prime}, b^{\prime \prime}, c^{\prime \prime}$ in modo da soddisfare ai tre sistemi :

$$
\begin{aligned}
& \frac{\partial^{2} x}{\partial u^{2}}=a \frac{\partial X}{\partial u}+b \frac{\partial X}{\partial v}+c X, \quad \frac{\partial^{2} x}{\partial u \partial v}=a^{\prime} \frac{\partial X}{\partial u}+b^{\prime} \frac{\partial X}{\partial v}+c^{\prime} X, \frac{\partial^{2} x}{\partial v^{2}}=a^{\prime \prime} \frac{\partial X}{\partial u}+b^{\prime \prime} \frac{\partial X}{\partial v}+c^{\prime \prime} X, \\
& \frac{\partial^{2} y}{\partial u^{2}}=a \frac{\partial Y}{\partial u}+b \frac{\partial Y}{\partial v}+c Y, \quad \frac{\partial^{2} y}{\partial u \partial v}=a^{\prime} \frac{\partial Y}{\partial u}+b^{\prime} \frac{\partial Y}{\partial v}+c^{\prime} Y, \frac{\partial^{2} y}{\partial v^{2}}=a^{\prime \prime} \frac{\partial Y}{\partial u}+b^{\prime \prime} \frac{\partial Y}{\partial v}+c^{\prime \prime} Y, \\
& \frac{\partial^{2} z}{\partial u^{2}}=a \frac{\partial Z}{\partial u}+b \frac{\partial Z}{\partial v}+c Z, \quad \frac{\partial^{2} z}{\partial u \partial v}=a^{\prime} \frac{\partial Z}{\partial u}+b^{\prime} \frac{\partial Z}{\partial v}+c^{\prime} Z, \frac{\partial^{2} z}{\partial v^{2}}=a^{\prime \prime} \frac{\partial Z}{\partial u}+b^{\prime \prime} \frac{\partial Z}{\partial v}+c^{\prime \prime} Z,
\end{aligned}
$$

perchè tutti col determinante $\sqrt{E G-F^{2}}$ diverso da zero, Si otterranno poi effettivamente le espressioni di tali coefficienti o risolvendo i dati sistemi tenendo conto delle (5) e (7), o derivando le (2), od usando lo stesso procedimento tenuto per le (2). In qualunque modo si faccia si ottiene: 


$$
\begin{aligned}
& a=\frac{\partial \alpha}{\partial u}+\alpha\left\{\begin{array}{c}
1 \\
1
\end{array}\right\}+\beta\left\{\begin{array}{c}
12 \\
1
\end{array}\right\}+\gamma, \quad b=\frac{\partial \beta}{\partial u}+\alpha\left\{\begin{array}{cc}
1 & 1 \\
2
\end{array}\right\}+\beta\left\{\begin{array}{c}
12 \\
2
\end{array}\right\}, \\
& c=\frac{\partial \gamma}{\partial u}-\alpha E-\beta F=\frac{\partial \mathbf{A}}{\partial u}-e, \\
& a^{\prime}=\frac{\partial \alpha}{\partial v}+\alpha\left\{\begin{array}{c}
12 \\
1
\end{array}\right\}+\beta\left\{\begin{array}{c}
22 \\
1
\end{array}\right\}, \quad b^{\prime}=\frac{\partial \beta}{\partial v}+\alpha\left\{\begin{array}{c}
12 \\
2
\end{array}\right\}+\beta\left\{\begin{array}{c}
22 \\
2
\end{array}\right\}+\gamma, \\
& c^{\prime}=\frac{\partial \dot{\gamma}}{\partial v}-\alpha F-\beta G=\frac{\partial \mathbf{A}}{\partial v}-f^{\prime}, \\
& a^{\prime}=\frac{\partial \alpha_{1}}{\partial u}+\alpha_{1}\left\{\begin{array}{c}
1 \\
1 \\
1
\end{array}\right\}+\beta_{1}\left\{\begin{array}{c}
12 \\
1
\end{array}\right\}+\gamma_{1}, \quad b^{\prime}=\frac{\partial \beta_{1}}{\partial u}+\alpha_{1}\left\{\begin{array}{c}
11 \\
2
\end{array}\right\}+\beta_{1}\left\{\begin{array}{c}
12 \\
2
\end{array}\right\}, \\
& c^{\prime}=\frac{\partial \gamma_{1}}{\partial u}-\alpha_{1} E-\beta_{1} F^{\prime}=\frac{\partial \mathbf{B}}{\partial u}-f, \\
& a^{\prime \prime}=\frac{\partial \alpha_{1}}{\partial v}+\alpha_{1}\left\{\begin{array}{c}
12 \\
1
\end{array}\right\}+\beta_{1}\left\{\begin{array}{c}
22 \\
1
\end{array}\right\}, \quad b^{\prime \prime}=\frac{\partial \beta_{1}}{\partial v}+\alpha_{1}\left\{\begin{array}{c}
12 \\
2
\end{array}\right\}+\beta_{1}\left\{\begin{array}{c}
22 \\
2
\end{array}\right\}+\gamma_{1}, \\
& c^{\prime \prime}=\frac{\partial \gamma_{1}}{\partial v}-\alpha_{1} F-\beta_{1} G=\frac{\partial \mathbf{B}}{\partial v}-g .
\end{aligned}
$$

Le due espressioni per $a^{\prime}, b^{\prime}, c^{\prime}$ ci dànno di nuovo la (II').

Calcolate così le (11), e tenendo presente le $(2)$, le note espressioni :

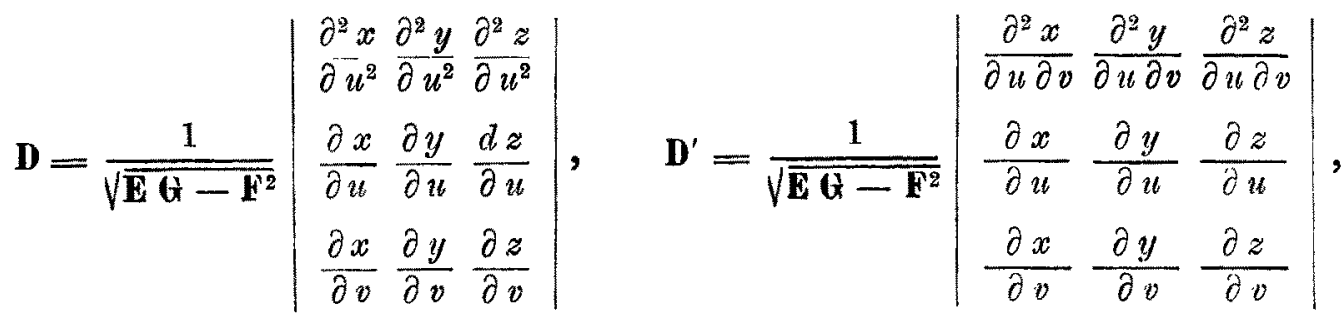

$$
\begin{aligned}
& \mathbf{D}^{\prime \prime}=\frac{1}{\sqrt{\mathbf{E G - \mathbf { F } ^ { 2 }}}}\left|\begin{array}{lll}
\frac{\partial^{2} x}{\partial v^{2}} & \frac{\partial^{2} y}{\partial v^{2}} & \frac{\partial^{2} z}{\partial v^{2}} \\
\frac{\partial x}{\partial u} & \frac{\partial y}{\partial u} & \frac{\partial z}{\partial u} \\
\frac{\partial x}{\partial v} & \frac{\partial y}{\partial v} & \frac{\partial z}{\partial v}
\end{array}\right|
\end{aligned}
$$


dànno :

$$
\begin{aligned}
& \mathbf{D}=\frac{\sqrt{E G-F^{2}}}{\sqrt{\mathbf{E G - \mathbf { F } ^ { 2 }}}}\left|\begin{array}{lll}
a & \alpha & \alpha_{1} \\
b & \beta & \beta_{1} \\
c & \gamma & \gamma_{1}
\end{array}\right|, \quad \mathbf{D}^{\prime}=\frac{\sqrt{E G-F^{2}}}{\sqrt{\mathbf{E}\left(-\mathbf{F}^{2}\right.}}\left|\begin{array}{ccc}
a^{\prime} & \alpha & \alpha_{1} \\
b^{\prime} & \beta & \beta_{1} \\
c^{\prime} & \gamma & \gamma_{\mathrm{s}}
\end{array}\right|,
\end{aligned}
$$

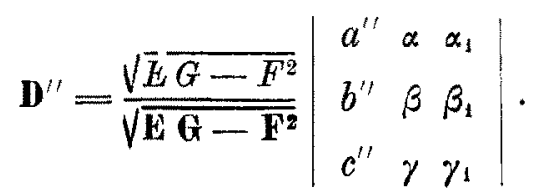

8. Teorema. Date due forme differenziali quadratiche (non proporzionali) di cui una almeno definita, si può, con una trasformazione reale delle variabili, ridurle ad avere proporzionali $i$ termini estremi, ed a mancare la definita del termine medio.

Per dimostrare tal teorema premettiamo un altro, che assieme alla dimostrazione togliamo dalla Geometria differenziale del Bıxcer. Alle due forme differenziali quadratiche non proporzionali :

$$
\begin{aligned}
& \Phi_{1}=a_{11} d x_{1}^{2}+2 a_{12} d x_{1} d x_{2}+a_{29} d x_{2}^{2}, \\
& \Phi_{2}=b_{11} d x_{1}^{2}+2 b_{12} d x_{1} d x_{2}+b_{22} d x_{2}^{2},
\end{aligned}
$$

di cui la prima sia per ipotesi definita, aggiungiamo la terza forma :

$$
\begin{aligned}
\Phi_{3} & =c_{11} d x_{1}^{2}+2 c_{12} d x_{1} d x_{z}+c_{22} d x_{2}^{2} \\
& =\frac{1}{\sqrt{a_{11} a_{22}-a_{12}^{2}}}\left|\begin{array}{ll}
a_{11} d x_{1}+a_{12} d x_{2} & a_{12} d x_{1}+a_{22} d x_{2} \\
b_{11} d x_{1}+b_{12} d x_{2} & b_{12} d x_{1}+b_{22} d x_{2}
\end{array}\right|,
\end{aligned}
$$

che è un covariante (irrazionale) simultaneo di $\Phi_{1}$ e $\Phi_{2}$. Il discriminante $c_{11} c_{32}-c_{12}^{2}$ si mette identicamente sotto la forma:

$$
\begin{gathered}
c_{11} c_{22}-c_{12}^{2}=\frac{-1}{a_{11} a_{22}-a_{12}^{2}}\left\{\left[\frac{a_{11} b_{22}-a_{22} b_{11}}{2}-\frac{a_{12}}{a_{11}}\left(a_{11} b_{12}-a_{12} b_{11}\right)\right]^{2}+\right. \\
\left.+\frac{a}{a_{11}^{2}}\left(a_{11} b_{12}-a_{12} b_{11}\right)^{2}\right\}
\end{gathered}
$$

perchè $a_{11}=0$, essendo $a_{11} a_{22}-a_{12}^{2}>0$. È dunque $c_{11} c_{22}-c_{12}^{2}<0$, e s'anmulla solo nel caso escluso della proporzionalità tra le due forme $\Phi_{1}$ e $\Phi_{2}$. Decomponiamo ora l'equazione differenziale quadratica $\Phi_{3}=0$ nei due fattori lineari distinti :

$$
\begin{aligned}
& \alpha d x_{1}+\beta d x_{2}=0, \\
& \gamma d x_{1}+\delta d x_{2}=0 .
\end{aligned}
$$


Siano $x_{1}^{\prime}=$ cost., $x_{2}^{\prime}=$ cost., $\mathrm{i}$ rispettivi integrali di queste due equazioni, ed introducendo queste nuove váriabili $x_{1}^{\prime}, x_{2}^{\prime}$, le due forme $\Phi_{1}$ e $\Phi_{2}$ si riducono a mancare del termine medio, mentre la terza riducesi solo al termine medio. Si ha così il teorema:

Date due forme differenziali quadratiche $\Phi_{1} e \Phi_{2}$, di cui una almeno $\Phi_{1}$ definita, si puo con una trasformazione reale di variabili ridurle a mancare contemporaneamente del termine medio. Le nuove variabili da introdursi sono quelle che, egnagliate a costanti, dùnno gl'integrati dell'equazione $\Phi_{3}=0$.

Nel caso eseluso della proporzionalità delle due forme la riduzione può farsi in infiniti modi.

Ciò posto cominciamo dal notare che le due forme $\Phi_{1}$ e $\Phi_{3}$ non sono proporzionali. Se ora applichiamo ad esse il detto teorema costruendo una quarta forma $\Phi_{4}$ loro covariante (irrazionale) simultaneo, si ba, introducendo le variabili che uguagliate a costanti dànno gl' integrali di $\Phi_{A}=0$, che le due forme $\Phi_{1}$ e $\Phi_{3}$ si riducono a mancare contemporaneamente del termine medio. Perciò, indicando con un indice tutti gli clementi dopo la trasformazione, si ha che la forma:

$$
\Phi_{3}^{\prime}=\frac{1}{\sqrt{a_{11}^{\prime} a_{22}^{\prime}-a_{12}^{\prime 2}}}\left|\begin{array}{ll}
a_{11}^{\prime} d x_{1}^{\prime}+a_{12}^{\prime} d x_{2}^{\prime} & a_{12}^{\prime} d x_{1}^{\prime}+a_{22}^{\prime} d x_{2}^{\prime} \\
b_{11}^{\prime} d x_{1}^{\prime}+b_{12}^{\prime} d x_{2}^{\prime} & b_{12}^{\prime} d x_{1}^{\prime}+b_{22}^{\prime} d x_{2}^{\prime}
\end{array}\right|,
$$

deve ridursi ai soli termini estremi, mentre $a_{12}^{\prime}=0$. Sarà dunque :

ossia :

$$
a_{11}^{\prime} b_{22}^{\prime}-a_{22}^{\prime} b_{11}^{\prime}=0
$$

$$
\frac{a_{11}^{\prime}}{b_{11}^{\prime}}=\frac{a_{92}^{\prime}}{b_{22}^{\prime}}
$$

assieme ad $a_{12}^{\prime}=0$. Ciò dimostra il teorema. Anche qui la riduzione può farsi in infiniti modi nel caso escluso della proporzionalità delle due forme $\Phi_{1}$ e $\Phi_{2}$. Notiamo che la nuova forma $\Phi_{4}^{\prime}$ si riduce al solo termine medio.

Interpretando la $\Phi_{i}$ come la forma differenziale quadratica che dà il quadrato dell'elemento lineare sopra una superficie, si ha che il sistema doppio di linee $\left(x_{1}, x_{2}\right)$ riducesi sempre ad un sistema doppio ortogonale, tanto se s'introducono le variabili che uguagliate a costanti dànno gl' integrali di $\Phi_{3}=0$, tanto quelle che derivano dagl'integrali di $\Phi_{4}=0$. Vogliamo far vedere che questi due sistemi doppi ortogonali sono bisettori l'un dell'altro. Introducendo prima le variabili che si hanno integrando $\Phi_{3}=0$, la $\Phi_{4}$ si 
riduce a :

$$
\Phi_{4}=\frac{a_{11} b_{22}-a_{22} b_{11}}{a_{11} a_{22}}\left(a_{11} d x_{1}^{2}-a_{22} d x_{3}^{2}\right)
$$

Per introdurre ora le altre nuove variabili, siccome $\frac{a_{11} b_{22}-a_{22} b_{11}}{a_{11} a_{22}} \geq 0$ basta integrare:

$$
a_{11} d x_{1}^{2}-a_{22} d x_{2}^{2}=0,
$$

che si scinde nelle altre due:

$$
\sqrt{a_{11}} d x_{1}-\sqrt{a_{22}} d x_{2}=0, \quad \sqrt{a_{11}} d x_{1}+\sqrt{a_{22}} d x_{2}=0 .
$$

È ora dimostrato quanto abbiamo asserito, perchè la prima di queste rappresenta l'equazione differenziale delle bisettrici del sistema doppio ortogonale $\left(x_{1}, x_{2}\right)$, e la seconda quella delle loro traettorie ortogonali.

Analogamente al modo come abbiamo costruito le forme $\Phi_{3}$ e $\Phi_{4}$ continuiamo a costruirne ancora delle altre $\Phi_{5}, \Phi_{0}, \ldots, \Phi_{i}$. Introducendo ora le variabili che uguagliate a costanti dànno gl'integrali di $\Phi_{i}=0$, le due forme $\Phi_{1}^{\prime}$ e $\Phi_{i-1}^{\prime}$ mancheranno contemporaneamente del termine medio, mentre la $\Phi_{i-2}^{\prime}$ conserva il termine medio, ma avrà gli estremi proporzionali ai corrispondenti di $\Phi^{\prime}{ }_{1}$. 立 facile poi vedere che più specialmente i termini estremi di $\Phi_{i-2}^{\prime}$ sono nulli, come quelli di $\omega^{\prime}{ }_{i}$. Siamo ricondotti al caso di $\Phi_{i}$, cioè gl'integrali di $\Phi_{i}=0$ sono anche integrali di $\Phi_{i-2}=0$. Conseguentemente $\Phi_{i-3}^{\prime}$ mancherà del termine medio, mentre $\Phi_{i-4}^{\prime}$ degli estremi; così proseguendo si vede che mentre $\Phi^{\prime}$, manca sempre del termine medio, la $\Phi_{2}^{\prime}$ avrà gli estremi proporzionali ai corrispondenti di $\Phi^{\prime}{ }_{1}$, oppure nullo il termine medio secondo che $i$ è pari o dispari.

9. Applicando il teorema del paragrafo precedente alle prime due forme differenziali quadratiche relative ad una data congruenza, si ha che con un'unica trasformazione reale di variabili si può sempre ottenere:

$$
F=0, \quad \frac{e}{E}=\frac{g}{G}=t
$$

ove $t \grave{\mathrm{e}}$ una certa fanzione di $u$ e $v$. Poniamo ora:

$$
x_{0}=x-t X, \quad y_{0}=y-t Y, \quad z_{0}=z-t Z,
$$

e riferiamo la congruenza a questa nuova superficie $S_{0}$ unica e determinata per qualunque congruenza.

I nuovi coefficienti della seconda forma diventano:

$$
e_{0}=e-t E=0, \quad f_{0}=f, \quad f_{0}^{\prime}=f^{\prime}, \quad g_{c}=g-t G=0 \text {. }
$$


Ritornando ora all'antica segnatura si ha che legando la congruenza a questa speciale superficie $S$, ed introducendo le nuove variabili $u$ e $v$ le prime due forme differenziali quadratiche assumono la forma:

$$
\begin{aligned}
d \sigma^{2}=\Sigma d X^{2} & =E d u^{2}+G d v^{2} \\
\Sigma d X d x & =\left(f+f^{\prime}\right) d u d v .
\end{aligned}
$$

Notiamo che tale riduzione è possibile sempre, ed in un sol modo, eccetto il caso in cui le due forme sono proporzionali, perehè allora è possibile in infiniti modi, e si ha $f+f^{\prime}=0$. Considereremo a parte tal caso. Indicando con $d p$ la lunghezza infinitesima della minima distanza del raggio $(u, v)$ dal raggio $(u+d u, v+d v)$, e con $r$ l'ascissa del suo punto d'incontro col raggio $(u, v)$, si ha in generale:

$$
\begin{gathered}
d p=\frac{1}{\sqrt{E G-F^{2}} d \sigma}\left|\begin{array}{cc}
E d u+F d v & F d u+G d v \\
e d u+f d v & f^{\prime} d u+g d v
\end{array}\right|, \\
r=\frac{-\Sigma d X d x}{\Sigma d X^{2}}=-\frac{e d u^{2}+\left(f+f^{\prime}\right) d u d v+g d v^{2}}{E d u^{2}+2 F d u d v+G d v^{2}} .
\end{gathered}
$$

Dopo le riduzioni precedenti queste diventano:

$$
d p=\frac{1}{\sqrt{E G} d \sigma}\left|\begin{array}{ll}
E d u & G d v \\
f d v & f^{\prime} d u
\end{array}\right|, \quad r=-\frac{\Sigma d X d x}{\Sigma d X^{2}}=-\frac{\left(f+f^{\prime}\right) d u d v}{E d u^{2}+G d v^{2}} .
$$

亡 $r=0$ solo sulle linee coordinate, percio il sistema doppio di rigate $(u, v)$ è tale che per ogni raggio i due punti centrali coincidono in $S$, e taglia perciò $S$ le linee di stringimento, ossia seconde linee che corrispondono per ortogonalità di elementi al sistema doppio ortogonale $(u, v)$ sulla sfera. Fissato il senso positivo di due rigate qualsiasi uscenti da un raggio $g$, si dirà angolo delle due rigate quello delle minime distanze di $g$ alle due generatrici successive sulle rispettive rigate percorse nel senso positivo. Intenderemo per verso di una rigata uscente da $g$ il senso secondo cui la generatrice successiva $g$ ' tende a girare intorno a $g$. Nell'immagine sferica l'angolo di due rigate si conserva o si cambia nel supplementare secondo che sono dello stesso verso, o di verso contrario. I piani passanti per $g$ e perpendicolari a quelle minime distanze s'intenderanno percorsi positivamente nel senso positivo delle relative immagini. Ad una rigata uscente da $g$ corrisponde un piano uscente da $g$, e reciprocamente per una determinata congruenza; un tal piano assume anche il nome della relativa rigata. 
Per una rigata qualsiasi definita dall' angolo $\theta$, è sulla immagine sferica $\operatorname{sen} \theta \cos \theta=\frac{\sqrt{E G} d u d v}{E d u^{2}+G d v^{2}}$, ossia la formola di HamiLton:

$$
r=-\frac{f+f^{\prime}}{2 \sqrt{E G}} \operatorname{sen} 2 \theta
$$

Questa ci dice che per due direzioni ortogonali qualunque $r$ ha valori uguali e disegno contrario, mentre ha il valore nullo per le rigate medie $u=$ cost., $v=$ cost., ed il valore massimo per le rigate principali, bisettrici di queste, e relative alle direzioni $\theta=+\frac{\pi}{4}, \theta=-\frac{\pi}{4}$. Adunque su ogni raggio un segmento di lunghezza $2 l=\frac{-\left(f+f^{\prime}\right)}{\sqrt{E G}}$ racchiude tutti i punti centrali: gli estremi di questo, punti limiti descrivono le due superficie limiti, mentre il punto medio genera la superficie media $S$. Evidentemente per un'osservazione fatta al paragrafo precedente le rigate principali sono quelle che s'ottengono introducendo le variabili che uguagliate a costanti dànno gl'integrali di $\Phi_{3}=0$.

Per due direzioni qualsiasi $\theta$ e $\frac{\pi}{2}-\theta r$ ha lo stesso valore: dette congiunte le rigate relative a tali direzioni, si ha che due rigate congiunte hanno, come le medie, le rigate principali per bisettrici. $\grave{\mathrm{E}} \omega=\frac{\pi}{2}-2 \theta$ l'angolo di due rigate congiunte, e si hanno come caso limite le rigate principali o le medie secondo che è $w$ nullo od uguale ad un retto Ci sono due sistemi doppi congiunti, ciò̀ formati di rigate congiunte, relativi allo stesso angolo $\omega$, e si passa dall'uno all'altro mediante una rotazione di un angolo retto. Le linee di stringimento di un sistema doppio congiunto qualsiasi $\left(u_{1}, v_{1}\right)$ stanno sopra una stessa superficie $S_{1}$. Se $S_{2}$ è la superficie relativa al sistema doppio congiunto $\left(u_{2}, v_{2}\right)$ ortogonale al precedente, si ha che queste due superficie sono in ogni istante equidistanti dalla media. I sistemi doppi di linee $\left(u_{1}, v_{1}\right),\left(u_{2}, v_{2}\right)$ corrispondono per ortogunalità di elementi alle immagini sferiche delle relative rigate congiunte. Perciò se è dato sopra una superficie un sistema doppio di linee $(u, v)$ che corrisponde per ortogonalità di elementi ad un sistema dopuio sulla sfera, si ha, conducendo per la data superficie le rette parallele alle normali nei punti corrispondenti della sfera, una congruenza in cui il sistema doppio $(u, v)$ è congiunto. Si avranno le rigate medie solo se il sistęma doppio sulla sfera era ortogonale. Per ulteriori sviluppi vedere il seguito di una mia nota cominciata ad apparire nel Giornale di Matematiche di Batraglin. 
Nel caso escluso della proporzionalità delle due forme è $f+f^{\prime}=0$, ossia $r=0$. Tutti $\mathrm{i}$ punti centrali coincidono nel punto medio e tutte le rigate sono medie. Simili congruenze segnalate da Ribadcotr diconsi isotrope. Si ha subito la proprietà caratteristica: La superficie media di una congruenza isotropa corrisponde per ortogonalità di elementi allimmagine sferica della congruenza.

Si hanno rigate sviluppabili quando è $d p=0$, ossia quando è:

$$
\frac{d u^{2}}{d v^{2}}=\frac{f}{f^{\prime}} \cdot \frac{G}{E}
$$

Essendo positivo il rapporto $\frac{G}{E}$, si avranno due sviluppabili reali od immaginarie secondo che $f$ ed $f^{\prime \prime}$ sono dello stesso segno o di segno contrario. Intanto $f$ ed $f^{\prime}$ sono ugualmente proporzionali ai coseni degli angoli che le linee, $u$ e $v$ su $S$ formano con le linee $v$ ed $u$ dell'immagine, perciò $f$ ed $f^{\prime}$ saranno dello stesso segno. se tali angoli sono entrambi superiori od inferiori ad un retto, di segno opposto nel caso contrario. In altri termini le superficie sviluppabili saranno immaginarie o reali serondo che le rigate medie hanno o no lo stesso verso.

In generale è sulla sfera:

$$
\frac{d u^{2}}{d v^{2}}=\frac{G}{E} \frac{\cos ^{2} \theta}{\operatorname{sen}^{2} \theta}
$$

perciò :

$$
\frac{f}{f^{\prime}}=\cot ^{2} \theta, \quad \operatorname{sen} 2 \theta=\frac{2 \sqrt{f f^{\prime}}}{f+f^{\prime}}
$$

e le due rigate sviluppabili corrispondono al doppio segno di $\theta$. I relativi punti centrali, fuochi, simmetrici rispetto al punto medio, e sempre nel segmento dei punti limiti, distano da questo di $\delta^{\prime}=-\frac{\sqrt{f f^{\prime}}}{f+f^{\prime}}$. L'angolo $2 \theta=\gamma$ dei rispettivi piani focali è dato da sen $y=\frac{\delta}{l}$.

Ciascuna rigata sviluppabile è ortogonale alla congiunta dell' altra. La superficie generata dei due fuochi $F_{1}$ ed $F_{z}$ è detta superficie focale; consta quindi di due falde: $S_{1}$ ed $S_{2}$. Considerando una falda $S_{1}$ abbiamo che una famiglia di rigate sviluppabili $u_{1}$ inviluppa un sistema di linee $u_{s}$ (caustiche) osculate dai relativi piani focali. Su essa le linee $v$, sono le linee di stringimento delle rigate congiunte alle sviluppabili $u_{1}$. Ora l'elemento $d v_{\mathrm{t}}$ sull'im- 
magine dovendo essere ortogonale al corrispondente $d v_{1}$ su $S_{4}$, ed al raggio passato per $F_{1}$, che ̀̀ tangente ad $S_{1}$ sarà necessariamente perpendicolare al piano tangente ad $S_{1}$. Onde le rigate $v_{\text {s }}$ tagliano ortogonalmente la falda $S_{1}$ della superficie focale, la quale ammetterà come piano tangente l'altro piano focale. Adunque l'inclinazione della normale ad $S_{1}$ sulla normale principale alla caustica uguaglia la mutua inclinazione dei piani congiunti; mentre l'inclinazione dei piani focali è uguale a quella della normale principale sul piano tangente.

Solo per le congruenze normali è $f=f$ : onde le superficie focali sono reali e coincidono con le superficie limiti, i piani focali sono ortogonali, e le caustiche sono geodetiche della superficie focale. 